\title{
Displacement monitoring using distributed macro-strain measurement
}

\author{
Yongsheng Tang \\ School of Urban Rail Transportation, Soochow University, Jixue Road 8\#, Xiangcheng District, \\ Suzhou 215137, China \\ E-mail: ystang@suda.edu.cn
}

Keywords: displacement monitoring; distributed macro-strain; long-gauge optical fiber sensor.

Abstract: As a key parameter the displacement is often monitored to assess the structural performance. However, it is not easy to implement the long-term monitoring under traffic loads especially. A new method is proposed to monitor displacement of flexural structures, based on the distributed macro-strain sensing technique. Within this method the distributed macro-strain was first obtained using long-gauge fiber Bragg grating (FBG) sensors. Then the classical displacement analysis theory, namely force method from the principle of virtual work, was applied to calculate the displacemnet. Finally a cantilever beam installed with the proposed long-gauge FBG sensors was subjected to free vibration to implement the verification. The results have verified the high accuracy of displacement measurement with the proposed method as well as high robustness to noise. Considering the other advantages of distributed optical fiber sensing, the proposed method presents broad application propects especially in long-term structural health monitoring (SHM).

\section{Introduction}

The factors, such as overloading, original structural flaws and steel corrusion, jeopardize the structures safety. Structural health monitoring (SHM) was proposed as a solution to ensure structural safety. Displacement is a key parameter to assess the beam structure performance, usually required for monitoring. However, it is not easy to accurately obtain displacements especially under traffic loads for long-term monitoring.

There are already many ways for displacement measurement. As a traditional method, displacement transducers is usually appiled to measure the dispalcement in the laboratory and filed tests. However, it is obviously not fit for long-term SHM due to its additional scaffolding requirements to support the displacement transducers. To solve the problem, Carlos et.al [1] developed a novel displacement transducer to measure vertical bridge deflections based on a liquid leveling system. However, it is of no use in dynamic monitoring as the liquid system's lag is too great. The global positioning system (GPS) has been developed and applicated in the last two decades, especially for large-scale bridge displacement monitoring [2,3]. However, GPS is limited by measuremnet accuracy, multi-path and cycle slips, a relatively low frequency of data, and the need for good satellite coverage. Another technique with lasers is also popular [4,5], but these types of laser sensors are not suitable for long-term SHM as they are often placed on the ground underneath the bridge and cannot be left unattended. Some other proposals have also been tried, such as photogrammetric deflection measurements [6], radar-based displacement sensors [7] and accelerometers [8]. However, it is still difficult to accurately implement the long-term displacement monitoring for most types of bridge.

In this paper, a new method is proposed by using the measured distributed strain to calculate the displacement. The theory of assessing displacment from strain is simple as only some basic structure mechanics is enough. Therefore, the most important part is the distributed strain sensing technology. In this paper the sensing methods is first introduced, and then the displacement assessing theory. Finally, the performance of the proposed method is verified with a cantilever beam. 


\section{Distributed strain measurements using long-gauge sensors}

The strain distribution is often not easy obtained with the traditional strain gauge. However, the displacement assessment will not be accurately implemented without the strain distribution. As shown in Fig. 1, if the average strain of each element can be measured for the beam, the displacement can also be evaluated. Here the average strain is called as macro-strain. Thereforce, it is vital to develop some technology for measuring the macro-strain.

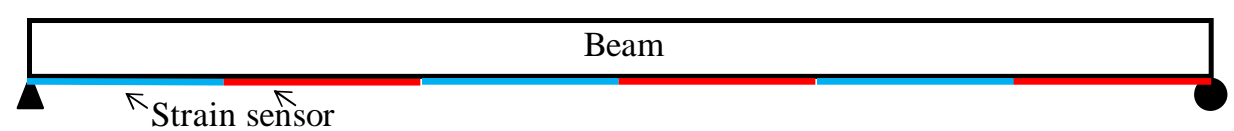

Fig. 1. Distributed strain measurement for beam.

Fiber Bragg grating (FBG) sensors have attracted attention for their use in SHM due to their excellent accuracy, high data acquisition speed and multiplexing performance. Based on the FBG technique, a long-gauge macro-strain sensor is developed at every point in the gauge length with identical mechanical behavior, and hence, the strain transferred from a shift of the Bragg center wavelength represents the average strain over the gauge length. As shown in Fig. 2, the FBG sensor is fixed at two ends within a plastic tube surrounded by a fiber sheath that is impregnated with epoxy resin. The diameter of the packaged sensor is approximately $1 \mathrm{~mm}$, while the gauge length can be set from 0.1 $\mathrm{m}$ to $2 \mathrm{~m}$.

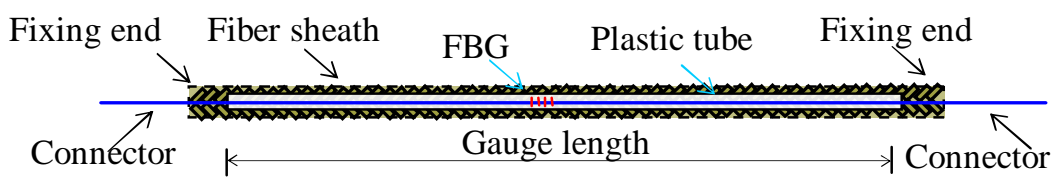

Fig. 2. Packaged long-gauge FBG sensor.

\section{Assessing displacement from distributed strain measurements}

It is well-known that the displacement can be easily calculated with actual strain distribution $\varepsilon(x)$ multiplying by the virtual strain distribution $\bar{\varepsilon}(x)$ from the principle of virtual work, as shown in Eq. 1 . Considering the case of macro-strain, the displacement $v$ can be expressed by Eq. 2 .

$$
\begin{gathered}
v=\int \frac{\varepsilon(x)^{*} \bar{\varepsilon}(x)}{E I} d x \\
v=\sum_{i=1}^{N} \frac{\varepsilon_{i} \bar{\varepsilon}_{i} L_{i}}{(E I)_{i}}
\end{gathered}
$$

where $\varepsilon_{i}$ and $\bar{\varepsilon}_{i}$ are the macro-strain for the $i_{t h}$ monitored element under actual loads and virtual unit force as shown in Fig. 3, respectively. $L_{i}$ is the $i_{t h}$ monitored element length. $(E I)_{i}$ is the bending stiffness of the $i_{\text {th }}$ monitored element. Therefore, after obtaining the distributed macro-strain, the displacement can be assessed with Eq. 2 for any location. 


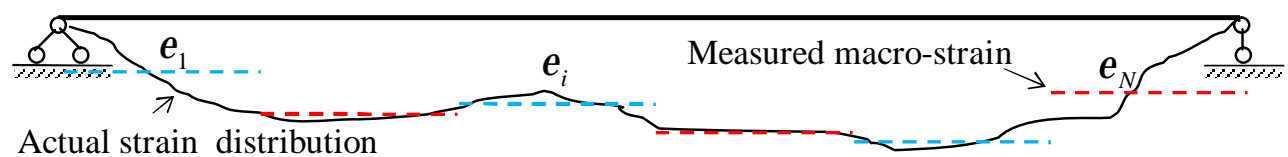

(a)

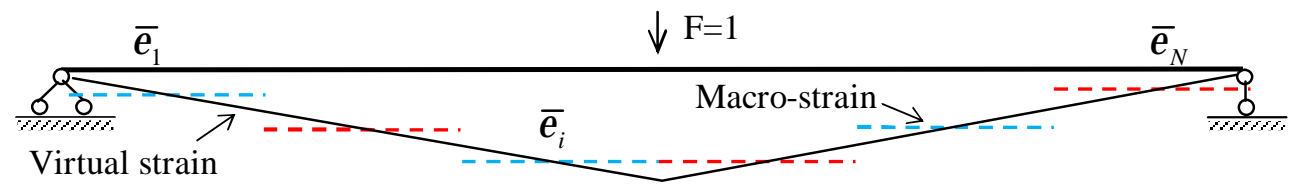

(b)

Fig. 3. Strain distribution under: (a) acutal loads and (b) virtual unit force.

\section{Verification with a cantilever beam test}

\section{Experiment setup}

A cantilever beam was applied to implement the dynamic tests with a depth of $7 \mathrm{~mm}$, a with of $150 \mathrm{~mm}$ and a length of $750 \mathrm{~mm}$. 5 long-gauge FBG sensors (see in Fig. 2) were distirbutedly installed on the down surface as shown in Fig. 4. For comparison, a displacement transducer was installed at node $N_{5}$ to obtain the true displacement value. Some displacement value was preset at node $N_{5}$ to cause free vibration. The sampling frequency was $1000 \mathrm{~Hz}$ with the FBG data logger.

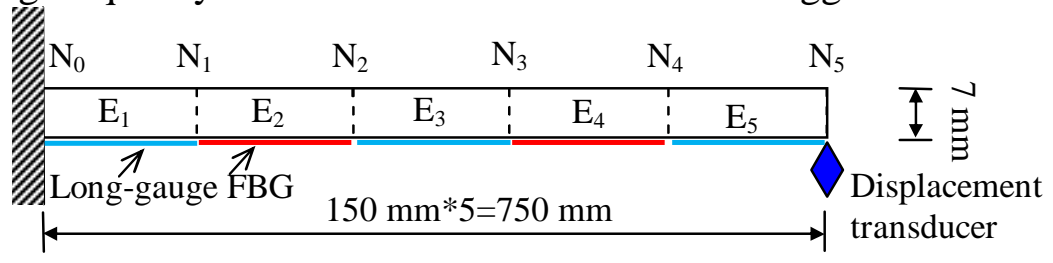

Fig. 4. Experiment setup (view from the side).

\section{Results and analysis}

Typical macro-strain results are as shown in Fig. 5 for the element $E_{1}$. Taking the strain distribution into Eq. 2, the displacement can be easily calculated for each node. However, only the results at node $N_{5}$ were included in this paper to investigate the performance of the proposed method, shown in Fig. 6. The conclusion is clear that the displacements assessed with the proposed method presenst high accuracy as they are close to the results of the displacement transducer. Some additional conclusion can also be found from the results that the displacement transducer measurement is easier effected by the noise than the proposed method.

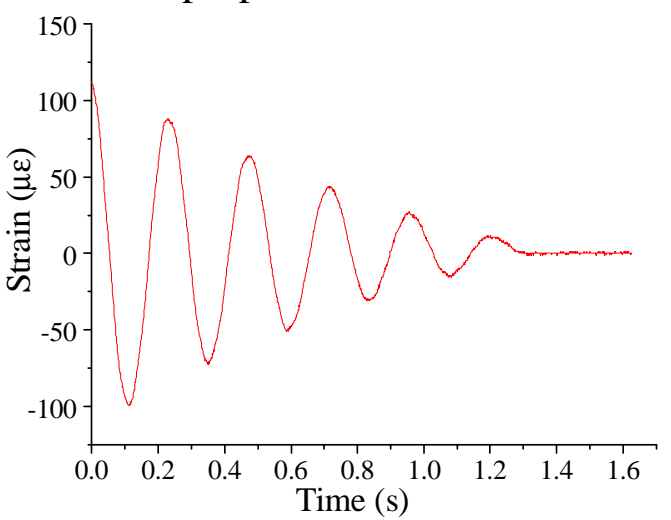

Fig. 5. Typical macro-strain results (E1).

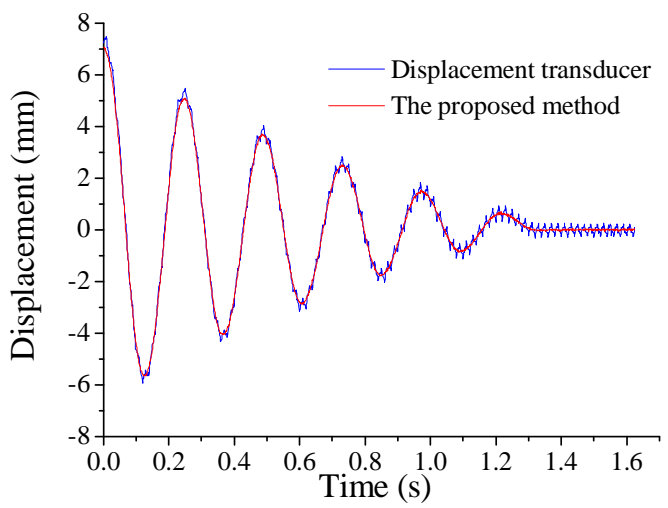

Fig. 6. Displacement results $\left(N_{5}\right)$. 


\section{Conclusions}

In this paper, a new method is proposed by using the measured distributed strain to calculate the displacement. The distributed strain methods is first introduced, and then the classical displacement analysis theory is developed with distrbuted macro-strain measurement. Finally a dynamic test was implemented with a cantilever beam to verify the proposed method. Therefore, the following conclusions can be drawn that the proposed method can be applied to accurately evaluate dynamic displacements. Combined with the advantages of fiber optic sensing, such as excellent performance under noise, the proposed method presents broad application propects especially in long-term SHM.

\section{Acknowledgements}

This paper is financially supported by University Natural Science Foundation of Jiangsu Province (14KJB580009), National Natural Science Foundation of China (51508364), Natural Science Foundation of Jiangsu Province (BK20150333) and Soochow University Foundation (SDY2014A10).

\section{References}

[1] R. Carlos, F. Carlos and F. Joaquim: Fiber-optic-based Displacement Transducer to Measure Bridge Deflections, Structural Health Monitoring, 10 (2011), p.147- p.156.

[2] J.W. Lovse, W.F. Teskey, G. Lachapelle and M.E. Cannon: Dynamic Deformation Monitoring of Tall StructureUsing GPS Technology, Journal of Surveying Engineering, 121 (1995), p.35- p.40.

[3] W.R. Gethin, X.L. Meng and H.D. Alan: Integrating a Global Positioning System and Accelerometers to Monitor the Deflection of Bridges, Journal of Surveying Engineering, 130 (2004), p.65- p.72.

[4] H.N. Hani, G. Mayrai and D. Joe: Comparison of Laser Doppler Vibrometer with Contact Sensors for Monitoring Bridge Deflection and Vibration, NDT\&E International, 38 (2005), p.213- p.218.

[5] M. Hyun, L. Seungmok and L. Bumjoo: Paired Structured Light for Structural Health Monitoring Robot System, Structural Health Monitoring, 10 (2011), p.49- p.64.

[6] J. Ruinian and V.J. David: Development of a Digital Close-range Photogrammetric Bridge Deflection Measurement System, Measurement, 43 (2010), p.1431- p.1438.

[7] A.R. Jennifer, C.Z. Li, C.Z. Gu and C.H. Justin: A Wireless Multifunctional Radar-based Displacement Sensor for Structural Health Monitoring, Proc. of SPIE, 7981 (2011), p.79810K1p.79810K11.

[8] K.T. Park, Kim SH, Park HS and Lee KW: The Determination of Bridge Displacement Using Measured Acceleration, Engineering Structures, 27 (2005), p.371- p.378. 